\title{
Remanent polarization of evaporated films of vinylidene fluoride oligomers
}

\section{AUTHOR(S):}

Noda, Kei; Ishida, Kenji; Kubono, Atsushi; Horiuchi, Toshihisa; Yamada, Hirofumi; Matsushige, Kazumi

\section{CITATION:}

Noda, Kei ...[et al]. Remanent polarization of evaporated films of vinylidene fluoride oligomers. JOURNAL OF APPLIED PHYSICS 2003, 93(5): 2866-2870

\section{ISSUE DATE:}

2003-03-01

URL:

http://hdl.handle.net/2433/39704

\section{RIGHT:}

Copyright 2003 American Institute of Physics. This article may be downloaded for personal use only. Any other use requires prior permission of the author and the American Institute of Physics. 


\title{
Remanent polarization of evaporated films of vinylidene fluoride oligomers
}

\author{
Kei Noda ${ }^{a}$ \\ Department of Electronic Science and Engineering, Kyoto University, Sakyo-ku, Kyoto 606-8501, Japan
}

Kenji Ishida

PRESTO, Japan Science and Technology Corporation (JST), and Department of Electronic Science and Engineering, Kyoto University, Sakyo-ku, Kyoto 606-8501, Japan

Atsushi Kubono

Department of Polymer Science and Engineering, Kyoto Institute of Technology, Sakyo-ku, Kyoto 606-8585, Japan

Toshihisa Horiuchi, Hirofumi Yamada, and Kazumi Matsushige

Department of Electronic Science and Engineering, Kyoto University, Sakyo-ku, Kyoto 606-8501, Japan

(Received 9 September 2002; accepted 4 December 2002)

A remanent polarization of $130 \pm 3 \mathrm{mC} / \mathrm{m}^{2}$, large among the values reported for organic materials, and rectangular $D-E$ hysteresis curves were realized in synthesized vinylidene fluoride (VDF) oligomer $\left[\mathrm{CF}_{3}\left(\mathrm{CH}_{2} \mathrm{CF}_{2}\right)_{17} \mathrm{I}\right]$ film evaporated onto a platinum surface around liquid nitrogen temperature. The results suggested that the VDF oligomer film has an extremely high crystallinity, and the electric dipoles arrange almost perfectly perpendicular to the film surface, and that a Lorentz local field factor of ferroelectric VDF oligomer crystals is nearly zero. Moreover, the obtained value of the coercive field, which was larger than those of ferroelectric polymers, might be attributed to the steric hindrance arising from the existence of iodine atoms at the VDF oligomer chains. (C) 2003 American Institute of Physics. [DOI: 10.1063/1.1540231]

\section{INTRODUCTION}

Ferroelectric polymers such as poly(vinylidene fluoride) $[\mathrm{PVDF}]^{1-3}$ and its copolymer $[\mathrm{P}(\mathrm{VDF} / \mathrm{TrFE})]^{4-12}$ have been well studied because of their applicability to transducers, sensors, actuators, and high density memories. With this background, elucidation of ferroelectric domain structures and the origin of ferroelectricity of these polymers is one of the important issues in terms of academic and practical points of view, leading to the realization of "molecular electronics" by controlling the arrangement of a small number of polar molecules. Highly ordered ultrathin films are necessary to examine this issue. However, it is hard to fabricate well-ordered polymer films with a nanoscale thickness because polymers naturally have mixed structures of both crystalline and amorphous phases. Therefore, we have focused on the vinylidene fluoride (VDF) oligomer ${ }^{13-17}$ and investigated ferroelectric characteristics of the VDF oligomer as well as the control of crystalline structures and molecular orientations by the vacuum evaporation method. The VDF oligomer $\left[\mathrm{CF}_{3}\left(\mathrm{CH}_{2} \mathrm{CF}_{2}\right)_{17} \mathrm{I}\right]$ has mainly two crystalline phases, ferroelectric (form I) and nonferroelectric (form II) phases, as shown in Fig. 1. This molecule crystallizes in form I crystal on alkali halide substrates $(\mathrm{KBr}, \mathrm{KCl})$, accompanied by epitaxial growth of the VDF oligomer films with fourfold symmetries. ${ }^{15}$ In previous studies, we reported the ferroelectricity of the VDF oligomer, using scanning probe microscopy (SPM) and transfer method, that the VDF oligomer films on $\mathrm{KBr}$ substrates are moved onto metal electrodes, revealing that the VDF oligomer is promising for

\footnotetext{
${ }^{a)}$ Electronic mail: nodakei@piezo.kuee.kyoto-u.ac.jp
}

nanoscale rewritable memories. ${ }^{16,17}$ However, it is indispensable to obtain knowledge about the coercive field $\left(E_{c}\right)$ and remanent polarization $\left(P_{r}\right)$ of this newly synthesized VDF oligomer on a macroscopic scale, which must be standards for evaluating the ferroelectricity of this molecule on a nanometer scale. In this study, we attempted to prepare ferroelectric phase crystals directly on metal substrates at low temperatures and evaluated the ferroelectric properties of the VDF oligomer films, quantitatively.

\section{SAMPLE AND EXPERIMENT}

The VDF oligomer used in this work was newly synthesized by Daikin Kogyo Co., Ltd. employing the telomerization method. The VDF oligomer evaporated film with a thickness of $500 \mathrm{~nm}$ was prepared onto a 200-nm-thick platinum film deposited onto a Si wafer covered with 500-nmthick thermal $\mathrm{SiO}_{2}$. This platinum film was fabricated by radio frequency magnetron sputtering at room temperature. The deposition rate of the VDF oligomer was 0.2 to 0.3 $\mathrm{nm} / \mathrm{min}$. The thickness and deposition rate were controlled monitoring a quartz oscillator. The substrate temperature $\left(T_{s}\right)$ was fixed at $-160^{\circ} \mathrm{C}$ during evaporation under a vacuum of $10^{-4} \mathrm{~Pa}$. After the evaporation, the substrate temperature was gradually heated to room temperature in vacuum.

The observation of the molecular conformation and crystalline phases in the VDF oligomer film was conducted by Fourier transform infrared spectroscopy (FTIR), utilizing a JEOL JIR 7500EM. An infrared spectrum of the film was measured in air by reflection absorption spectroscopy (RAS) at an angle of incidence of $80^{\circ}$. 


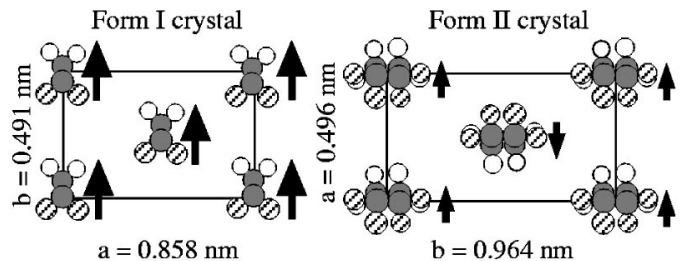

Form I (all-trans)

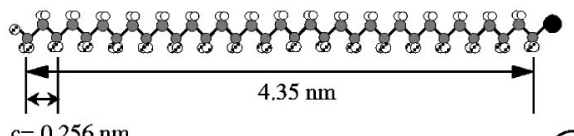

Form II (TGTG')

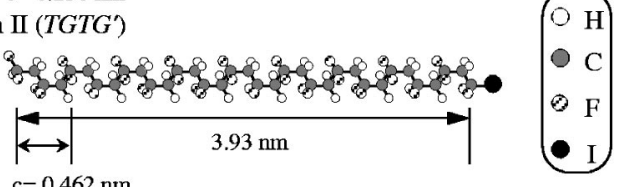

FIG. 1. Schematic diagrams of the crystalline structures and the molecular chains of the VDF oligomer. There are mainly two crystalline phases, form I (ferroelectric phase) and form II (nonferroelectric phase) crystals. The arrows show the direction of permanent electric dipole moments which exist because of the difference of electronegativities among hydrogen, carbon, and fluorine atoms.

After the IR measurement, the 40-nm-thick aluminum films as circular upper electrodes were fabricated onto the VDF oligomer film surface by shadow masking evaporation under a vacuum of $10^{-3} \mathrm{~Pa}$ at room temperature. The diameters of the $\mathrm{Al}$ electrodes were about $0.3,0.6$, and $1.1 \mathrm{~mm}$, respectively. The measurements of current-voltage $(I-V)$ characteristics and $D-E$ hysteresis curves of the VDF oligomer film were performed with a system for evaluating ferroelectrics, which consisted of a current/charge to voltage converter (Toyo Corporation Model 6252), an arbitrary waveform generator (Biomation 2414B), and an analog-to-digital converter (WaveBook 516). All measurements were carried out in air at room temperature.

\section{RESULTS AND DISCUSSION}

Figure 2 shows the IR RAS spectrum of the VDF oligomer film deposited at $T_{s}=-160{ }^{\circ} \mathrm{C}$. The absorption bands at 846, 890, 1200, and $1300 \mathrm{~cm}^{-1}$ strongly appeared in Fig. 2. As the bands at 846 and $890 \mathrm{~cm}^{-1}$ are characteristic of form I, it was revealed that form I crystals could be formed dominantly on any substrates around liquid nitrogen temperature. However, the appearance of somewhat broad bands at 1200 and $1300 \mathrm{~cm}^{-1}$ showed that the film consisted of both form I and form II crystals. The band at $1400 \mathrm{~cm}^{-1}$, assigned to the wagging vibration $\left[\mathrm{w}\left(\mathrm{CH}_{2}\right)\right]$, appeared weakly in the RAS spectrum. The small absorption of $\mathrm{w}\left(\mathrm{CH}_{2}\right)$ revealed that the $c$ axis (molecular axis) in the film was considerably parallel to the substrate. In PVDF, the fabrication of form I crystals during the evaporation process with low temperature substrates was reported before. ${ }^{18}$ Form I phase is also likely to appear during the crystallization from the melting PVDF with an ultraquenching technique. ${ }^{19}$ These previous papers indicate the crucial contribution of the quenching effects to the formation of ferroelectric phase crystals in the VDF oligomer film mentioned above.

Figure 3 presents the current-voltage characteristic of

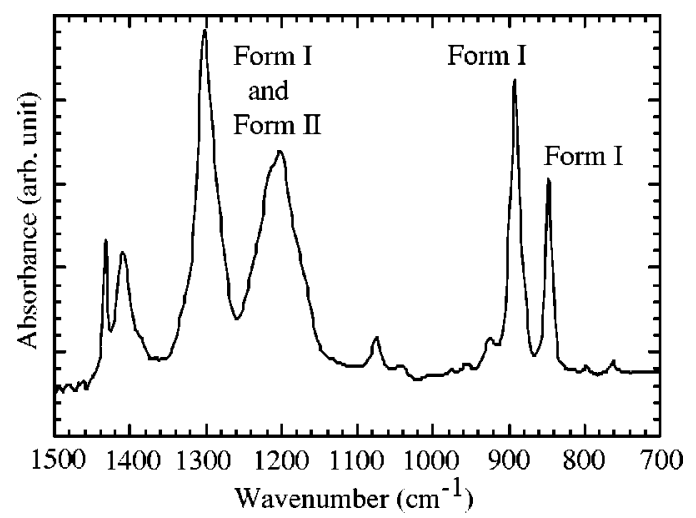

FIG. 2. FTIR RAS spectrum of the VDF oligomer film fabricated at $-160{ }^{\circ} \mathrm{C}$ on a platinum substrate by the evaporation method.

the VDF oligomer film. The amplitude and frequency of a triangular wave voltage applied between upper and lower electrodes were $120 \mathrm{~V}$ and $0.015 \mathrm{~Hz}$, respectively. In Fig. 3, narrow peaks appeared at $\pm 60 \mathrm{~V}$, accompanied by nonlinear electric conduction in the regions of higher electric fields. These peaks obviously indicated the polarization reversal which was owing to $180^{\circ}$ rotation of the VDF oligomer molecules in the direction of the applied voltage. The coercive field of the 500-nm-thick VDF oligomer film was estimated to be $120 \mathrm{MV} / \mathrm{m}$, according to the positions of the sharp peaks in the $I-V$ curve observed here. This value of $E_{c}$ was about twice as much as those of $\mathrm{PVDF}^{2}$ and $\mathrm{P}(\mathrm{VDF} / \mathrm{TrFE}),{ }^{5,7}$ which are about $50 \mathrm{MV} / \mathrm{m}$.

Next, we calculated the value of the remanent polarization $\left(P_{r}\right)$ of the VDF oligomer film from the integral of one of the peaks observed in Fig. 3, which corresponds to $2 P_{r}$. However, the conduction current flowing in the background of the $I-V$ curve should be removed beforehand to estimate $P_{r}$. Figure 4 shows the magnification of the measured $I-V$ curve [Fig. 4(a)], the curve fitted for the background of the measured $I-V$ curve [Fig. 4(b)], and the result of the subtraction of the latter from the former curve [as shown in Fig. 4(c)]. The curve of Fig. 4(b) could be exactly expressed by

$$
I_{b}=\frac{\epsilon \epsilon_{0} S}{l} \frac{d V}{d t}+m_{1} \sinh \left(m_{2} V\right),
$$

where $I_{b}$ is the current in the background, $\epsilon$ the dielectric constant of the film (hereafter $\epsilon \simeq 10$ was experimentally obtained), $\epsilon_{0}$ the permittivity of free space, $S$ the area of the upper electrode, $l$ the film thickness, and both $m_{1}$ and $m_{2}$ are positive fitting parameters, respectively. The first term in the right side of Eq. (1) stands for the dielectric displacement current of the film. The second term obeys an ionic conduction which is caused by the impurities included in the film during the evaporation. These impurities are probably iodized compounds which are the residues remaining after the synthesis of the VDF oligomer.

A rectangular $D-E$ hysteresis curve of the VDF oligomer film was produced from the integral of the corrected $I-V$ curve [Fig. 4(c)], as shown in Fig. 5. The value of $P_{r}$ was calculated to be $130 \pm 3 \mathrm{mC} / \mathrm{m}^{2}$, which revealed a con- 


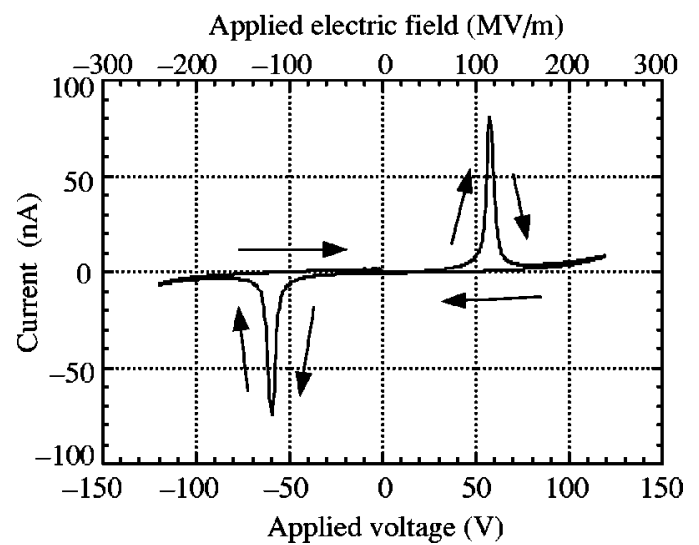

FIG. 3. Polarization switching current of the VDF oligomer film with a thickness of $500 \mathrm{~nm}$ on the platinum substrate. Two peaks appear at $\pm 60 \mathrm{~V}$ due to the $180^{\circ}$ rotation of the VDF oligomer molecules induced by applied triangular wave voltage, revealing the ferroelectricity of the VDF oligomer.

siderably larger value compared to experimental values of PVDF(about $\left.60 \mathrm{mC} / \mathrm{m}^{2}\right)^{2}$ and $\mathrm{P}(\mathrm{VDF} / \mathrm{TrFE}$ ) (about $\left.100 \mathrm{mC} / \mathrm{m}^{2}\right){ }^{6,8,21}$

A theory which gives $P_{r}$ of ferroelectric polymer films containing both crystalline and amorphous phases was established by Wada. ${ }^{20} \mathrm{He}$ assumed that the polar crystal is an ellipsoid with the depolarization coefficient $\left(L_{m}\right)$ and led the following equation,

$$
P_{r}=\phi\left[\frac{\epsilon}{\epsilon+L_{m}\left(\epsilon_{c}-\epsilon\right)}\right] P_{\mathrm{sc}} \psi=\phi f P_{\mathrm{sc}} \psi,
$$

where $\epsilon$ is the dielectric constant of the film, $\epsilon_{c}$ the dielectric constant of crystal, $P_{\mathrm{sc}}$ the spontaneous polarization of a single form I crystal, $\phi$ the volume fraction of crystals in the film, and $\psi$ the degree of orientation of dipole moments of the crystal along the thickness direction.

$P_{\text {sc }}$ was theoretically calculated with a variety of methods. $^{21-25}$ If it is assumed that the monomer units of PVDF are rigid electric dipoles of $7.0 \times 10^{-30} \mathrm{Cm}$ and all of the dipoles are aligned like in the form I crystal, $P_{\mathrm{sc}}$ would be $130 \mathrm{mC} / \mathrm{m}^{2}$ which is hereafter defined as $P_{0}$. In fact, the effect of the local electric field on the polarizable molecules must be considered in the case of predicting $P_{\mathrm{sc}} \cdot{ }^{21} P_{\mathrm{sc}}$ is given as

$$
P_{\mathrm{sc}}=\left[1+L_{c}\left(\epsilon_{\infty}-1\right)\right] P_{0},
$$

where $L_{c}$ and $\epsilon_{\infty}$ are the Lorentz factor and the highfrequency dielectric constant, respectively. Broadhurst et al. utilized an Onsager cavity method, which is often applied to the ensemble of molecules in liquids, and the Lorentz local field. As a result, $P_{\mathrm{sc}}$ of PVDF was expected to be $220 \mathrm{mC} / \mathrm{m}^{2} .{ }^{22}$ However, the Lorentz local field does not suit the case for form I crystal of PVDF, which belongs to an orthorhombic structure. Al-Jishi and Taylor took both the local field and the orthorhombic system of PVDF into consideration. ${ }^{23}$ Besides, the approach that the dipoles are replaced as not point dipoles but two point charges $( \pm q)$ seperated by a certain distance was introduced to their calculation scheme. ${ }^{23}$ They found that $P_{\text {sc }}$ and $L_{c}$ would be $127 \mathrm{mC} / \mathrm{m}^{2}$ and -0.007 , implying that the contribution of

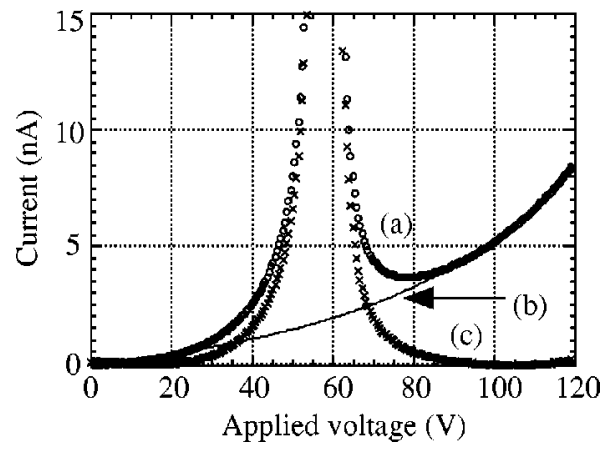

FIG. 4. Magnification of current-voltage characteristics (Fig. 3). (a) Measured $I-V$ curve. (b) The curve fitted for the background of the measured $I-V$ curve. (c) The result of the subtraction of the latter from the former curve. The value of $P_{r}$ was calculated by the integral of (c).

the local electric field on the amount of polarization would be negligible. ${ }^{24}$ Ogura and Chiba reported calculated values of the spontaneous polarization of ferroelectric polymers using the iteration method, ${ }^{25}$ which showed that $P_{\mathrm{sc}}$ of PVDF would be $128.7 \mathrm{mC} / \mathrm{m}^{2}$.

Here, we presume that $P_{\text {sc }}$ of the VDF oligomer is $130 \mathrm{mC} / \mathrm{m}^{2}$. According to Eq. (2) and the experimental value of $P_{r} \simeq 130 \mathrm{mC} / \mathrm{m}^{2}$, we get $\phi f \psi \simeq 1$. In general, the depolarization coefficient $\left(L_{m}\right)$ can be regarded as a small value in ferroelectric polymers. ${ }^{26}$ Then, we take $f \simeq 1$ and $\phi \psi \simeq 1$ is obtained. In short, $\phi \simeq 1$ and $\psi \simeq 1$ are simultaneously held good. This consideration proposes that the VDF oligomer film has only a crystalline region, and that the polarization axes ( $b$ axes) of the VDF oligomer form I crystals are oriented along the thickness direction after the poling process during the measurement of $D-E$ hysteresis curves. From the above-mentioned results, the VDF oligomer is expected to be one of the models for predicting ultimate activities and clarifying the origin of ferroelectric behaviors in fluorinated organic ferroelectrics.

Another way for the removal of the bad influence of the ionic conduction on the characterization of ferroelectric properties is increasing the frequency of the applied voltage up to the extent that the charge carriers in the film cannot follow in the change of the applied voltage. Figure 6 shows the $I-V$ curve [Fig. $6($ a) $]$ and $D-E$ hysteresis curve [Fig. 6(b)] of the VDF oligomer film, which were measured by applying the triangular wave with the frequency of $800 \mathrm{~Hz}$ and the amplitude of $160 \mathrm{~V}$. Under this condition, a hysteresis phenomenon was clearly observed without the appearence of the ionic conduction. Here, the values of $E_{c}$ and $P_{r}$ were measured to be $160 \mathrm{MV} / \mathrm{m}$ and $112 \mathrm{mC} / \mathrm{m}^{2}$, respectively. The larger $E_{c}$ and smaller $P_{r}$ than those acquired in the low frequency measurement implied that there existed VDF oligomer molecules which could not respond to the change of the applied voltage with the high frequency of 800 Hz. Nevertheless, $P_{r}$ of the VDF oligomer showed the value almost twice as large as that of PVDF. In Fig. 6(b), the measured hysteresis curve has a broad width and the spontaneous polarization $\left(P_{s}\right)$ is almost the same as the value of $P_{r}$. These characteristics reflect both an extremely high crystallinity and the perpendicular orientation of $b$ axes to the substrate in the VDF oligomer film. 


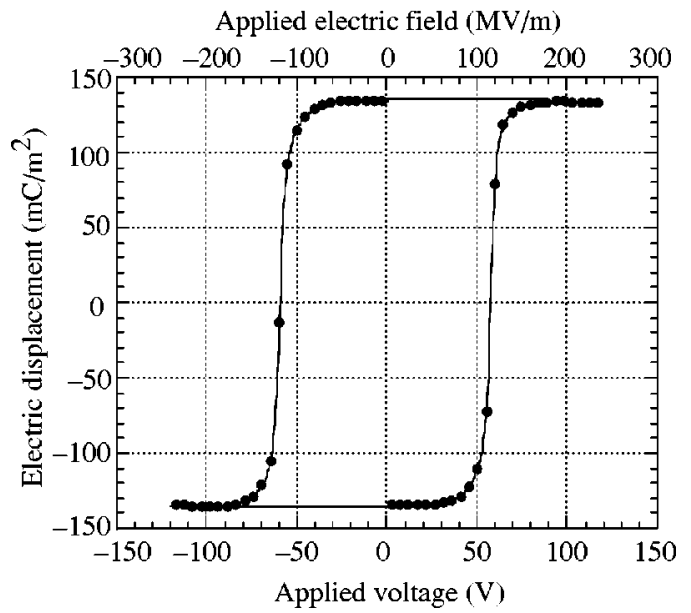

FIG. 5. $D-E$ hysteresis curves observed for the 500-nm-thick VDF oligomer film produced from the integral of the $I-V$ curve measured at a frequency of $0.015 \mathrm{~Hz}$.

Although both form I and form II crystals existed within the 500-nm-thick film in accordance with Fig. 2, this film displayed great activities as a ferroelectric material. It is possible that the phase transformation of form II to form I crystals might be brought about by applying high electric fields during the poling process of the film. The details of this speculation are under investigation.

The reason why the VDF oligomer owned a little larger value of the coercive field in comparison with other ferroelectric polymers might be ascribed to the excellent crystallinity of the VDF oligomer or the steric hindrance between molecules due to the existence of iodine atoms at the end of molecular chains. The steric hindrance is likely to restrict the molecular chain motions such as kink propagations ${ }^{27}$ or flipflop motions ${ }^{28}$ during the polarization reversal. A rectangular-shaped $D-E$ hysteresis curve with $E_{c}$ $=33-40 \mathrm{MV} / \mathrm{m}$ and $P_{r}=110 \mathrm{mC} / \mathrm{m}^{2}$ at room temperature was presented in single crystalline (SC) films of $\mathrm{P}(\mathrm{VDF} / \mathrm{TrFE}){ }^{29}$ This $E_{c}$ of SC films without any amorphous regions and lamellar crystals is slightly smaller than that of $\mathrm{P}(\mathrm{VDF} / \mathrm{TrFE})$ films reported in previous papers. ${ }^{5,7,21}$ The increase of crystallinity does not seem to be related to the increase of $E_{c}$ very much. In the present stage, taking this result into account, the steric hindrance between the VDF oligomer molecules caused by iodine atoms during the polarization reversal can be thought of as the main contribution to the large $E_{c}$ of the VDF oligomer.

So far the demonstration of the ferroelectricity in evaporated films of organic ferroelectrics has been rare on account of the difficulty in the formation of upper electrodes, which withstand application of high electric fields, onto surfaces of evaporated soft films. In the past study on the evaporated film of $\mathrm{P}(\mathrm{VDF} / \mathrm{TrFE})$, the value of $P_{r}$ obtained from the $D-E$ hysteresis measurement became much lower than those of the spin-cast films, which was attributed to an unfavorable morphology or texture of the vapor-deposited material. ${ }^{30}$ On the contrary, the VDF oligomer evaporated film has such a great function that the value of $P_{r}$ is much larger than that of ferroelectric polymers, suggesting that the
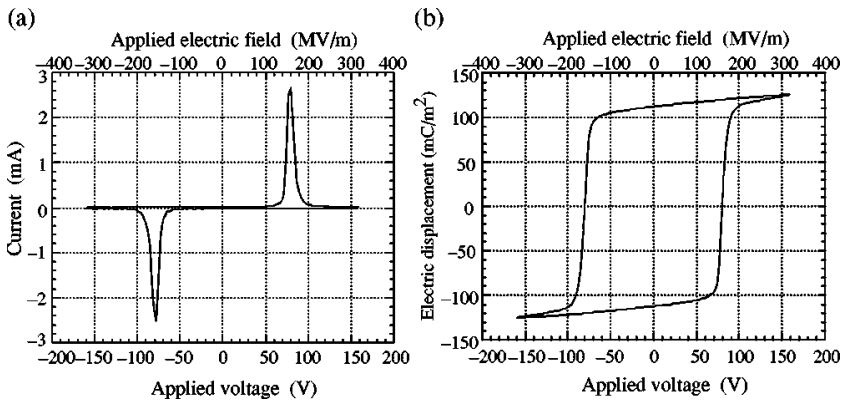

FIG. 6. (a) $I-V$ curve and (b) $D-E$ hysteresis curve of the 500-nm-thick VDF oligomer film directly observed at a frequency of $800 \mathrm{~Hz}$.

VDF oligomer used in this study is suitable for control of ferroelectric properties in thin films by the evaporation method.

\section{CONCLUSIONS}

In conclusion, we successfully observed the polarization switching current and the $D-E$ hysteresis curve for the VDF oligomer film directly deposited on a platinum substrate around liquid nitrogen temperature. These results assured the ferroelectricity of the newly synthesized VDF oligomer. Both the coercive field and remanent polarization measured in this study are larger than typical values of other organic ferroelectrics. Above all, high crystallinity of the VDF oligomer led to the supreme value of $P_{r}\left(130 \pm 3 \mathrm{mC} / \mathrm{m}^{2}\right)$. This article proposes that the VDF oligomer is one of the promising candidates for new generations of high quality ferroelectric devices which can be fabricated by dry processes.

\section{ACKNOWLEDGMENTS}

The authors thank Daikin Kogyo Co., Ltd. for providing the VDF oligomer. We express our gratitude to Dr. H. Ohigashi for fruitful discussions. The authors are also grateful to the support of the Kyoto University-Venture Business Laboratory and a Grant-in-Aid for Scientific Research from the Ministry of Education, Culture, Sports, Science and Technology of Japan. K. N. gratefully acknowledges Research Fellowships of the Japan Society for the Promotion of Science for Young Scientists.

${ }^{1}$ H. Kawai, Jpn. J. Appl. Phys. 8, 975 (1969).

${ }^{2}$ T. Furukawa, M. Date, and E. Fukada, J. Appl. Phys. 51, 1135 (1980).

${ }^{3}$ A. J. Lovinger, Science 220, 1115 (1983).

${ }^{4}$ K. Tashiro and M. Kobayashi, Phase Transitions 18, 213 (1989).

${ }^{5}$ G. T. Davis, M. G. Broadhurst, A. J. Lovinger, and T. Furukawa, Ferroelectrics 57, 73 (1984).

${ }^{6}$ T. Furukawa, IEEE Trans. Electr. Insul. 24, 375 (1989).

${ }^{7}$ K. Kimura and H. Ohigashi, Jpn. J. Appl. Phys., Part 1 25, 383 (1986).

${ }^{8}$ H. Ohigashi, K. Omote, and T. Gomyo, Appl. Phys. Lett. 66, 3281 (1995).

${ }^{9}$ A. V. Bune, V. M. Fridkin, S. Ducharme, L. M. Blinov, S. P. Palto, A. V. Solokin, S. G. Yudin, and A. Zlatkin, Nature (London) 391, 874 (1998).

${ }^{10}$ Q. M. Zhang, V. Bharti, and X. Zhao, Science 280, 2101 (1998).

${ }^{11}$ X. Chen, H. Yamada, T. Horiuchi, and K. Matsushige, Jpn. J. Appl. Phys., Part 1 37, 3834 (1998).

${ }^{12}$ T. Fukuma, K. Kobayashi, T. Horiuchi, H. Yamada, and K. Matsushige, Thin Solid Films 397, 133 (2001). 
${ }^{13}$ Herman, S. Umemoto, T. Kikutani, and N. Okui, Polym. J. (Tokyo) 30, 659 (1998)

${ }^{14}$ Y. Yoshida, K. Ishida, K. Ishizaki, T. Horiuchi, and K. Matsushige, Jpn. J. Appl. Phys., Part 1 36, 7389 (1997).

${ }^{15}$ K. Noda, K. Ishida, A. Kubono, T. Horiuchi, and K. Matsushige, J. Appl. Phys. 86, 3688 (1999).

${ }^{16}$ K. Noda, K. Ishida, A. Kubono, T. Horiuchi, H. Yamada, and K. Matsushige, Jpn. J. Appl. Phys., Part 1 39, 6358 (2000).

${ }^{17}$ K. Noda, K. Ishida, A. Kubono, T. Horiuchi, H. Yamada, and K. Matsushige, Jpn. J. Appl. Phys., Part 1 40, 4361 (2001).

${ }^{18}$ A. Takeno, N. Okui, T. Kitoh, M. Muraoka, S. Umemoto, and T. Sakai, Thin Solid Films 202, 205 (1991).

${ }^{19}$ C. C. Hsu and P. H. Geil, J. Appl. Phys. 56, 2404 (1984).

${ }^{20}$ Y. Wada, Ferroelectrics 57, 393 (1984).

${ }^{21}$ Y. Tajitsu, H. Ogura, A. Chiba, and T. Furukawa, Jpn. J. Appl. Phys., Part 1 26, 554 (1987).
${ }^{22}$ M. G. Broadhurst, G. T. Davis, J. E. McKinney, and R. E. Collins, J. Appl. Phys. 49, 4992 (1978).

${ }^{23}$ R. Al-Jishi and P. L. Taylor, J. Appl. Phys. 57, 897 (1985).

${ }^{24}$ R. Al-Jishi and P. L. Taylor, J. Appl. Phys. 57, 902 (1985).

${ }^{25}$ H. Ogura and A. Chiba, Ferroelectrics 74, 347 (1987).

${ }^{26}$ S. Ikeda, M. Jimbo, S. Kobayashi, and Y. Wada, J. Polym. Sci., Polym. Phys. Ed. 23, 1781 (1985).

${ }^{27}$ H. Dvey-Aharon, T. J. Sluckin, P. L. Taylor, and A. J. Hopfinger, Phys. Rev. B 21, 3700 (1980).

${ }^{28}$ H. Ohigashi, K. Omote, H. Abe, and K. Koga, J. Phys. Soc. Jpn. 68, 1824 (1999).

${ }^{29}$ H. Ohigashi, Mater. Res. Soc. Symp. Proc. 600, 23 (2000).

${ }^{30}$ C. Fischer, J. K. Krüger, K.-P. Bohn, U. Vogt, J. Schreiber, R. Jiménez, D. Wolf, J. F. Legrand, P. Alnot, and B. Servet, J. Polym. Sci., Part B: Polym. Phys. 33, 237 (1995). 